\title{
EDITORIAL
}

\section{And the winner should be...}

\author{
It is time that the tremendous contribution made by Carl Woese to microbiology, medicine \\ and biology as a whole is rewarded by the Nobel committee.
}

Among microbiologists, Carl Woese's achievements are well known. His most lauded accomplishments include the recognition of an entirely new domain of organisms, the Archaea ${ }^{1}$, and the subsequent introduction of the three-domain phylogeny ${ }^{2}$ that is now widely recognized as the most accurate reflection of the relatedness of all organisms. We are so used to using neatly organized taxonomic trees to demonstrate the relationships between different microorganisms that it is easy to forget that not so long ago the classification of bacteria was considered hopeless. In 1963 Roger Stanier, then one of the foremost researchers in the field of bacterial classification, proclaimed that "The ultimate scientific goal of biological classification cannot be achieved in the case of bacteria" (REF. 3). At that time, the classification of organisms relied on shared phenotypes, but bacteria often have too few to allow clear categorization on this basis. It was Woese who first suggested that genetic information, and in particular the sequence of the $16 \mathrm{~S}$ ribosomal RNA gene (which had been shown previously to be highly conserved), could be used to classify organisms. 16S rRNA analysis revealed that the methanogens, which Woese investigated at the suggestion of his colleague Ralph Wolfe, are not related to any known bacteria, and this was the first step in the discovery of the Archaea ${ }^{1}$. Ultimately, Woese demonstrated that all cellular life can be divided into three domains - Bacteria, Archaea and Eukarya - a tremendous shift from the five-kingdom classification that was prevalent previously.

Carl Woese's accomplishments have been recognized by various organizations worldwide, and his awards include a MacArthur Foundation grant, the prestigious Leeuwenhoek medal (awarded by the Dutch Royal Academy of Sciences) and election to the US National Academy of Sciences. However, one prize that he has not been awarded, despite receiving several nominations, is the Nobel Prize. In part, this reflects the fact that his accomplishments may seem to be a difficult fit for the prize in physiology or medicine (and even more so for the prizes in physics and chemistry). Indeed, there are no known pathogenic archaea, and the biggest initial benefit of $16 \mathrm{~S}$ rRNA-based phylogenetic analysis was seen in microbial ecology, as it allowed many unculturable organisms to be identified through sequencing alone. However, 16S rRNA-based phylogenetic trees are increasingly leading to advances in medicine, primarily in two ways. First, direct sequencing of the $16 \mathrm{~S}$ rRNA genes in patient isolates can allow rapid disease diagnosis. As culturing is not required, sequence analysis can be used to diagnose many different infectious diseases and is now common in clinical diagnostic laboratories. In the case of Whipple's disease, it even led to the identification of a previously unknown bacterium (Tropheryma whipplei) as the cause ${ }^{4}$. Second, using $16 \mathrm{~S}$ rRNA-based phylogeny, it is possible to study the human microbiota at a detailed level. In recent years, we have begun to obtain a comprehensive picture of the composition of the human-associated microbiota in various niches, including the gut, skin and oral cavity. This has led to the insight that shifts in the gut-associated microbiota are associated with diseases such as Crohn's disease and inflammatory bowel disease, and has allowed us to study how the gut microbiota changes during ageing and in response to eating or antibiotics. We are only at the beginning of our investigations into the human-associated microbiota, but it is already clear that these organisms play a crucial part in our health and well-being. In the future, an improved understanding of the microbiota may allow us to diagnose diseases on the basis of microbiota composition and to design specific interventions.

Carl Woese has completely changed the way we view the relationships between all organisms on Earth, revealed the presence of a previously unrecognized domain and provided us with a tool that has begun to elucidate the complex composition of the human microbiome, which constitutes $90 \%$ of the genetic diversity of our bodies and has been called the second human genome $e^{5}$. It is difficult to think of more-fundamental discoveries that are affecting the way we think about the environment and human health alike. As the attentions of the scientific community turn once again to the decisions of the Nobel committee, perhaps it is time to campaign for Carl Woese to receive the recognition that he deserves.

\footnotetext{
Woese, C. R. \& Fox, G. E. Phylogenetic structure of the prokaryotic domain: the primary kingdoms. Proc. Natl Acad. Sci. USA 74, 5088-5090 (1977).

2. Woese, C. R., Kandler, O. \& Wheelis, M. L., Towards a natural system of organisms: proposal for the domains Archaea, Bacteria, and Eucarya. Proc. Natl Acad. Sci. 87, 4576-4579 (1990).

3. Stanier, R. Y., Doudoroff, M. \& Adelberg, E. A. The Microbial World 2nd edn (Prentice-Hall, Englewood Cliffs, 1963).

4. Relman, D. A., Schmidt, T. M., MacDermott, R. P. and, Falkow, S Identification of the uncultured bacillus of Whipple's disease. N. Engl. J. Med. 327, 293-301 (1992).

5. Relman, D. A. \& Falkow, S. The meaning and impact of the human genome sequence for microbiology. Trends Microbiol. 9, 206-208 (2001).
} 